\title{
Predictors of fatigue following the onset of infectious mononucleosis
}

\author{
B. CANDY, T. CHALDER, A. J. CLEARE, A. PEAKMAN, A. SKOWERA,
} S. WESSELY, J. WEINMAN, M. ZUCKERMAN AND M. HOTOPF ${ }^{1}$

From the Department of Psychological Medicine, Department of Immunology, Public Health Laboratory and Medical Microbiology, Guy's, King's and St Thomas' School of Medicine and Institute of Psychiatry, London

\begin{abstract}
Background. Infectious mononucleosis (IM) is a risk factor for chronic fatigue. Reduced activity is the most consistent factor found to be associated with poor outcome following the onset of infectious mononucleosis. However, little is known about the biological mechanisms involved in the pathogenesis of chronic fatigue following IM and no study, so far, has examined the relation between certain illness beliefs and poor outcome. This study explored immunological, endocrine, behavioural and cognitive responses to the acute illness and assessed which components of these groups of risk factors predicted a chronic course.
\end{abstract}

Method. Using a prospective cohort design, 71 primary care patients with IM were enrolled onto the study and interviewed. Their recovery was explored by postal questionnaire up to 1 year later.

Results. In the univariate analysis, increased baseline levels of immune activation were associated with fatigue at baseline and 3 months. Cortisol levels were not associated with fatigue at any point. Using multivariate models of clinical and psychosocial baseline factors, severity of symptoms and illness perceptions were found to predict fatigue 3 months later. At 6 months, fatigue was best predicted by female gender and illness perceptions, and at 12 months by female gender and a symptoms-disability factor.

Conclusions. In the multivariate analysis no factors were found to predict poor outcome at all timepoints. Instead the pattern of predictors changed over time, partly but not completely consistent with our a priori predictions. Larger studies are needed to explore further the predictive nature of biopsychosocial factors in the pathogenesis of chronic fatigue related to IM. The psycho-behavioural predictors found in this study are amenable to intervention. Such interventions should be tested in randomized controlled trials.

\section{INTRODUCTION}

There is substantial evidence that infectious mononucleosis (IM), often referred to as glandular fever, and other severe viral infections are risk factors for chronic fatigue (Berelowitz et al. 1995; Hotopf et al. 1996; Buchwald et al. 2000; White et al. 2001). White's cohort study demonstrated a five-fold increase in chronic fatigue among individuals suffering from IM compared

\footnotetext{
1 Address for correspondence: Dr Matthew Hotopf, Department of Psychological Medicine, Guy's King's and St Thomas' School of Medicine, 103 Denmark Hill, London SE5 8AZ.
}

with controls that had suffered upper respiratory tract infections (White et al. 1998). There are a number of possible mechanisms that could explain this association. Psycho-behavioural mechanisms have been postulated for fatigue following severe infections, and there is some evidence that individuals who have a history of previous psychiatric disorder (Hotopf et al. 1996); physical attributional style (Cope et al. 1994); greater adverse life events (Buchwald et al. 2000); greater family support (Buchwald et al. 2000); and lower fitness (White et al. 2001) are at greater risk of poorer outcomes. In a 
recent systematic review the most consistent factor found for delayed recovery in IM was reduced physical functioning (Candy et al. 2003). Thus, these cognitive and behavioural factors might predict a pattern of response that leads to deconditioning, which is an important candidate for perpetuating fatigue. More distal predictors of an individual's response to illness may also be important, and we have recently shown that unexplained symptoms in adulthood are associated with childhood experiences of illness (Hotopf et al. 1999, 2000).

There are also a range of important biological candidate mechanisms for the association between glandular fever and chronic fatigue. One possibility is the immune response associated with glandular fever, and White and colleagues (2001) found that acute fatigue was associated with a raised CD8 cell count. There is considerable evidence that immune function may be altered in chronic fatigue, but there is no consistent pattern of immune profile associated with the condition (Wessely et al. 1998). It is important, therefore, to determine whether immune changes that occur in illnesses that may precipitate chronic fatigue syndrome (CFS) contribute to subsequent fatigue.

CFS is also associated with alterations in neuroendocrine function, with a relative under activity of the hypothalamic-pituitary-adrenal axis (HPA) (Cleare et al. 2001; Parker et al. 2001). While this seems a robust finding in individuals with established CFS, without prospective studies of individuals at high risk of developing CFS it is difficult to tell whether such changes play a causal role, or are a result of downstream factors such as sleep, deconditioning or circadian rhythm disruption (Spath Schwalde et al. 1992; Stupnicki \& Obminski, 1992; Leese et al. 1996).

\section{Aims and hypothesis}

This study aimed to bring together diverse biological and psychological risk factors in studying the pathogenesis of chronic fatigue related to IM. The study tested the following hypotheses.

1 Increases in immune activation are associated with severity of acute fatigue. Such immune activation does not predict chronic fatigue.

2 Individuals with lower salivary cortisol at baseline would be at greater risk of developing chronic fatigue. Chronic fatigue at follow-up would also be associated with reduced salivary cortisol concentrations.

3 A range of behavioural and psychological factors at baseline will predict subsequent fatigue: these include childhood experience of chronic illness, physical attributional style, illness perceptions that perceived the illness as having serious consequences, and a reduction in activity.

\section{METHOD}

\section{Recruitment}

Potential participants were identified by positive IM serology from three haematology and two virology laboratories, and by six general practices and one student health centre. First contact with the patient was made by their GP and only on agreement were they approached and invited to participate by the researcher. If they agreed to participate they were asked for an interview in their own home as soon as possible. Patients were all invited to participate in a randomized controlled trial of a brief psycho-educational intervention, or usual care. The results of this randomized trial have been reported elsewhere (Candy et al. 2003). We aimed to gain a consecutive sample of all patients positive for IM from the laboratories. Efforts were made to remind clinicians in the general practices and student health centre about the study, but it is likely that a proportion of cases of IM will have been missed.

\section{Participants}

The inclusion criteria were: (1) aged between 16 and 45 years; (2) clinically diagnosed IM; and (3) serological evidence of recent IM either from a heterophile antibody test (Microgen Bioproducts Ltd, UK) or by using the VCA IgM immunofluorescence assay (Immuno concepts Inc, Sacramento, CA). Patients with IM were not eligible if they were reported by the GP to have additional physical illnesses that may be associated with fatigue (such as endocrine disorders). Patients with psychotic illnesses (schizophrenia and bipolar disorder) and substance misuse were also excluded, as were individuals who had limited literacy skills as most of the variables were measured using questionnaires. In practice this did not lead to any exclusions. 


\section{Baseline data}

Whole blood was collected (into EDTA) to measure activated CD4 helper and CD8 cytotoxic T cells. Activation was assessed by surface expression of the marker HLA-DR and measured by flow cytometry using specific monoclonal antibodies. Activated CD4 and CD8 T cells were measured as the percentage of each population co-expressing the class II HLA molecule HLA-DR, a well-recognized marker of chronically activated $\mathrm{T}$ cells.

Participants were asked to give four saliva samples on a single day using the salivette (Sarstedt, UK) collection system. Sample times were $08.00,12.00,16.00$ and $20.00 \mathrm{~h}$. Subjects were asked to not eat or drink anything in the hour before collection, and to sit or lie down for $20 \mathrm{~min}$ before they collected their sample. Women were also asked to collect their saliva only within the first week of their menstrual cycle. We measured the cortisol concentration in each sample to gain an overall measure of HPA axis activation across the day.

At baseline participants completed a range of questionnaires. Fatigue was measured on the Chalder Fatigue Questionnaire (Chalder et al. 1993) an 11-item fatigue scale. Each item consists of four possible answers that can either be scored $0 / 1$ or $0,1,2,3$. The former system is used to generate a case definition $(>3)$, the latter is used to generate a continuous fatigue score (0-33). Psychological distress was assessed on the 12-item General Health Questionnaire (GHQ12) (Goldberg \& Williams, 1988); functional status on the Work and Social Adjustment Scale (Marks, 1986) and the Short Form-36 (SF-36) (Garratt et al. 1993); social support on the Significant Others Scale (Power et al. 1988); life events on the Life Events Inventory (Cochrane \& Robertson, 1973); and sufferers' understanding and expectations of current illness on the Illness Perceptions Questionnaire (Weinman et al. 1996). Additional questions were asked on their childhood experience of chronic illness, their current symptoms and expectations of recovery and on any illness advice they were given by health professionals.

\section{Follow-up data}

Participants were also asked to supply saliva samples at 3 and 6 months. Postal questionnaires at 3 and 6 months repeated measures taken at baseline. Only the fatigue questionnaire was sent at 12 months.

\section{Statistical analysis}

The main measure of poor outcome was fatigue caseness, as defined as scoring $>3$ on the fatigue questionnaire (Chalder et al. 1993). We also used fatigue as a continuous variable measured on the same questionnaire.

In the first instance, baseline and follow-up fatigue symptoms (measured as a continuous variable) were correlated with baseline immune activation. Results were expressed as percentage activated CD4 or CD8 cells.

The main parameter used to indicate cortisol profiles at each time point was the total output calculated as area under the curve (AUC) using the trapezoidal method. In addition, we took four secondary measures: peak value (highest of the four samples), trough value (lowest of the four samples), mean value (mean of the four samples), and the diurnal change (peak minus trough). These measures were compared with those from a healthy control group of similar age (mean $=22.6$ years). In order to explore the possible relationship of these parameters with fatigue, we undertook cross sectional analyses at baseline, 3 months and 6 months, and a prospective analysis of whether baseline cortisol parameters were associated with fatigue at 3 and 6 months.

The relationships between psycho-behavioural baseline variables and fatigue caseness at baseline, 3, 6 and 12 months were determined using odds ratios and $95 \%$ confidence intervals. Logistic regression analysis was used to control for potential confounders. For each time point, we determined the relationship between baseline characteristics and fatigue. The analytical strategy we used was firstly to determine whether socio-demographic factors (age and gender), as well as treatment group, were associated with fatigue at each time point. All analyses were controlled for treatment group. If age or gender was not associated at $P<0 \cdot 2$ they were not included in subsequent analyses. The next step was to add baseline clinical characteristics (symptoms and disability/functionality), and again if these were not significantly associated they were not included further. Finally, illness perceptions 
were added and controlled for the effects of baseline clinical variables.

\section{RESULTS}

Seventy-one $(51 \%)$ of 139 referred primary care patients agreed to participate. None of the participants were excluded due to being over 45 years, having psychotic illness or substance misuse. The most common reason for non-participation was inaccurate contact details held by the laboratory or general practice. Participants were compared with non-participants on age, gender and location (inner city or not) of the general practice at which they were registered. This revealed similarity in age and practice location, but males were less likely to participate (39\% v. $54 \%)$. Of the 71 patients identified, all had a positive monospot test. EBV serology was available on 41 cases, in whom $36(88 \%)$ were positive for EBV IgM.

Sixty-nine per cent of those who participated responded to follow-up at 3 months, $87 \%$ at 6 months and $70 \%$ at 12 months. There were no difference in age, sex or baseline fatigue scores between responders and non-responders at 6 months. At 12 months, non-responders were more likely to be women $(P=0.09)$ and older $(P=0.09)$, but there were no differences in baseline fatigue status. Due to technical difficulties blood samples were received for immunological analysis from $45 \%$ of the sample. Saliva samples for cortisol analysis were returned by $66 \%$ of participants.

\section{Baseline characteristics}

Characteristics of patients are shown in Table 1. The sample was generally young, just under half were students and most were single. The median time from onset of symptoms to first assessment was 38 days. There was no association between level of fatigue at baseline, and time between onset of symptoms and interview.

At baseline interview 41\%(29/71) reported that fatigue was their main IM symptom. Eightyfive per cent of participants, measured by the Fatigue Questionnaire (Chalder et al. 1994), were fatigue cases; $4 \%$ reported that fatigue had lasted $>6$ months and $68 \%$ reported not to have recovered. Table 2 shows the fatigue status at follow-up. Over time, fewer participants were fatigue cases, more had recovered, and more
Table 1. Characteristics of sample at baseline assessment

\begin{tabular}{ll}
\hline \hline Female & $60 \%$ \\
Mean age (s.D.) & $22 \cdot 9(8 \cdot 2)$ \\
Student & $46 \%$ \\
Living with parent or partner & $68 \%$ \\
History of emotional problems & $20 \%$ \\
Days from onset to first assessment & $38(\mathrm{IQR}: 29-60)$ \\
GP diagnosed IM at first consultation & $27 \%$ \\
Days off sick & $10(\mathrm{IQR}: 5-16)$ \\
GHQ score (s.D.) & $16 \cdot 7(6 \cdot 2)$ \\
Physical functioning SF-36 (s.D.) & $79 \cdot 6(22 \cdot 4)$ \\
Physical functioning in last month & $20 \cdot 8(34 \cdot 8)$ \\
SF-36 (s.D.) & $56 \cdot 5(42 \cdot 2)$ \\
Emotional functioning in last month & \\
SF-36 (s.D.) & $8 \cdot 8(8 \cdot 5)$ \\
Work and Social Adjustment Scale score (s.D.) & $24 \%$ \\
Believed recovery will take >1 month & $20 \cdot 3(3 \cdot 3)$ \\
IPQ control cure (s.D.) & $19 \cdot 2(4 \cdot 5)$ \\
IPQ consequences (s.D.) & $19 \cdot 9(5 \cdot 2)$ \\
Fatigue score (s.D.) & $85 \%$ \\
Case of fatigue & $4 \%$ \\
Fatigue for >6 months & $68 \%$ \\
Not recovered from IM & \\
\hline
\end{tabular}

Table 2. Fatigue status at follow-up

\begin{tabular}{|c|c|c|c|}
\hline & $\begin{array}{c}3 \text { months } \\
N=49\end{array}$ & $\begin{array}{c}6 \text { months } \\
N=62\end{array}$ & $\begin{array}{c}12 \text { months } \\
N=50\end{array}$ \\
\hline Fatigue score & $15 \cdot 5(5 \cdot 6)$ & $14 \cdot 1(4 \cdot 5)$ & $14 \cdot 1(5 \cdot 0)$ \\
\hline Fatigue case & $47 \%$ & $40 \%$ & $38 \%$ \\
\hline $\begin{array}{l}\text { Fatigue lasting } \\
>6 \text { months }\end{array}$ & $14 \%$ & $11 \%$ & $5 \%$ \\
\hline $\begin{array}{l}\text { Not recovered } \\
\text { from IM }\end{array}$ & $45 \%$ & $38 \%$ & NA \\
\hline
\end{tabular}

NA, Not assessed.

reported that their fatigue had lasted $>6$ months.

\section{Univariate analysis}

\section{Immune activation}

During the acute phase (within 6 weeks of onset of IM) those defined as fatigue cases had a higher percentage of activated CD4 lymphocytes compared to those who were not cases, (median, $13 \cdot 2 \%$ v. 6.2, Mann-Whitney $U=9 \cdot 00$, $P=0 \cdot 025, N=21)$. There was a higher percentage of activated CD8 lymphocytes in fatigue cases compared with non-fatigue cases (median $53.8 \%$ v. $25.5 \%$; Mann-Whitney $U=7 \cdot 00$, $P=0 \cdot 016, N=21)$. Fatigue cases at 3 months also had higher acute phase percentages of activated CD4 (median $25.6 \%$ v. 9.9\%, MannWhitney $U=5 \cdot 0, P=0 \cdot 02, N=14)$. Similarly, 
fatigue cases at 3 months had higher activated CD8 counts at baseline compared with nonfatigue cases (median, $79 \cdot 1 \%$ v. $42 \cdot 1 \%$; MannWhitney $U=7 \cdot 00, P=0 \cdot 039, N=14)$. Fatigue at 6 months was also associated with higher baseline activation of CD4 and CD8 counts, but this difference did not reach statistical significance. Because of the small number of samples gained these factors were not explored in multivariate analysis.

\section{Cortisol measures}

There were no differences in any of the five cortisol parameters between fatigue cases and non-cases when analysed cross sectionally at baseline, 3 months and 6 months. The AUC values were $95 \cdot 5($ s.D. $31 \cdot 8) \mathrm{nmol} / \mathrm{l} / \mathrm{h}$ in cases and $90 \cdot 1$ (s.D. $14 \cdot 6) \mathrm{nmol} / 1 / \mathrm{h}$ in non-cases $(t=0 \cdot 34$, $P=0 \cdot 74)$ at baseline, $93 \cdot 8($ s.D. $31 \cdot 1) \mathrm{nmol} / \mathrm{l} / \mathrm{h}$ in cases and $79 \cdot 4$ (s.D. 27.4) $\mathrm{nmol} / \mathrm{l} / \mathrm{h}$ in non-cases (Mann-Whitney $U=97 \cdot 00, P=0 \cdot 35$ ) at 3 months and 88.8 (S.D. 24.2) nmol/1/h in cases and 83.4 (s.D. $39 \cdot 6) \mathrm{nmol} / \mathrm{l} / \mathrm{h}$ in non-cases $(t=0 \cdot 43, P=$ $0.67)$ at 6 months. Similarly, there were no significant correlations between fatigues scores and cortisol parameters at baseline, 3 or 6 months. We also categorized subjects into low or high AUC using the lowest AUC quartile to define a low cortisol state. Those with low AUC cortisol values had similar fatigue scores to those with high values at both baseline (low AUC, 20.2 (4.1) points; high AUC, 19.8 (5.5) points, $t=0 \cdot 20, P=0 \cdot 84$ ) and at 6 months (low AUC, $11.4(2 \cdot 8)$ points; high AUC, 14.4 (4.6) points, $t=1 \cdot 67, P=0 \cdot 1)$. Using the same analysis we compared baseline cortisol parameters for all participants, and for the subgroup who were defined as fatigue cases at baseline, with the control group. We found no significant differences.

In the prospective analysis, the change in AUC cortisol between baseline and 6 months was not related to fatigue caseness at 6 months. The mean change score for those with fatigue caseness at 6 months was $4 \cdot 7$ (s.D. 35.1) and in non-cases 8.9 (s.D. 40.9) (Mann-Whitney $U=$ $79 \cdot 5, P=0 \cdot 40)$. Those with low baseline AUC values did not differ in their fatigue scores at 6 months follow-up from those with high baseline AUC values (low AUC, 14.9 (5.7) points; high AUC, $13.9(4.3)$ points, $t=0.62, P=0.53)$.

\section{Behavioural and psychological factors}

Table 3 gives the univariate odds ratios and confidence intervals for the hypothesized variables. At baseline, lower socio-economic group, and clinical features including having worse symptoms, lower physical functioning, and a higher general health questionnaire (GHQ) score were associated with fatigue caseness. By 3 months, fatigue caseness was predicted by a history of emotional problems, clinical features, a longer convalescence, baseline fatigue score, a belief that their recovery will take more than 1 month and that IM would have serious consequences on their life. At 6 months being female, lower functionality and a belief at baseline that IM would have more serious consequences on their life (illness perception questionnaire (IPQ) consequences subscale) predicted fatigue caseness. Baseline functionality, and level of symptoms were associated with fatigue caseness 12 months later.

\section{Multivariate models}

We used logistic regression analyses to model the effects of independent variables on fatigue cases. Because there were a wide range of potential baseline variables measuring symptoms and disability, we used a varimax factor analysis to reduce these variables to a smaller number of factors. The variables entered into the analysis were baseline total symptom score (i.e. a measure of the severity of the acute symptoms of glandular fever), GHQ score, SF36 scores (three subscales), Work and Social Adjustment Scale (WSAS), and total fatigue score. The analysis yielded a two factor solution (see Table 4). Factor one (symptoms-disability) was loaded heavily by total physical symptoms, physical functioning on the SF36 and WSAS score. Factor two (fatigue-depression) comprised total fatigue and GHQ scores at baseline. These factors were standardized to generate a mean of 0 and a standard deviation of 1 .

Table 5 shows the results of the logistic regression analysis. At 3 months, the strongest predictors of fatigue were total symptoms at baseline and the belief that they will take $>1$ month to recover from IM. At 6 months, female gender was associated with fatigue, as were illness perceptions (both the beliefs that the illness would take $>1$ month to recover from 
Table 3. Univariate odds ratios $(95 \%$ CI) for baseline associations with fatigue at four time points

\begin{tabular}{|c|c|c|c|c|}
\hline$N$ fatigue cases $/ N$ completed assessment & $\begin{array}{c}\text { Baseline } \\
60 / 71\end{array}$ & $\begin{array}{c}3 \text { months } \\
23 / 49\end{array}$ & $\begin{array}{c}6 \text { months } \\
25 / 62\end{array}$ & $\begin{array}{c}12 \text { months } \\
19 / 50\end{array}$ \\
\hline \multicolumn{5}{|l|}{ Sociodemographic features } \\
\hline Female gender & $2 \cdot 1(0 \cdot 6-7 \cdot 6)$ & $2 \cdot 0(0 \cdot 6-6 \cdot 4)$ & $3 \cdot 8(1 \cdot 3-12 \cdot 3)$ & $2 \cdot 7(0 \cdot 7-10 \cdot 6)$ \\
\hline Student & $0 \cdot 4(0 \cdot 2-1 \cdot 7)$ & $0 \cdot 9(0 \cdot 3-2 \cdot 8)$ & $0 \cdot 8(0 \cdot 3-2 \cdot 3)$ & $0 \cdot 8(0 \cdot 3-2 \cdot 7)$ \\
\hline Age $>19$ years* & $0 \cdot 5(0 \cdot 1-2 \cdot 0)$ & $0 \cdot 6(0 \cdot 2-2 \cdot 0)$ & $0 \cdot 9(0 \cdot 3-2 \cdot 4)$ & $0 \cdot 5(0 \cdot 2-1 \cdot 7)$ \\
\hline Non-manual occupation & $0 \cdot 2(0 \cdot 4-0 \cdot 9)$ & $0 \cdot 4(0 \cdot 1-1 \cdot 4)$ & $0 \cdot 9(0 \cdot 3-2 \cdot 4)$ & $1 \cdot 2(0 \cdot 4-3 \cdot 8)$ \\
\hline Lives with parent or partner & $0 \cdot 8(0 \cdot 2-3 \cdot 1)$ & $0 \cdot 5(0 \cdot 1-1 \cdot 6)$ & $0 \cdot 6(0 \cdot 2-1 \cdot 8)$ & $0 \cdot 4(0 \cdot 1-1 \cdot 4)$ \\
\hline More than one recent adverse life event & $3 \cdot 9(0 \cdot 8-19 \cdot 8)$ & $1 \cdot 5(0 \cdot 5-4 \cdot 6)$ & $1 \cdot 2(0 \cdot 4-3 \cdot 4)$ & $2 \cdot 4(0 \cdot 7-7 \cdot 7)$ \\
\hline Childhood experience of illness in family & $0 \cdot 6(0 \cdot 2-2 \cdot 1)$ & $1 \cdot 8(0 \cdot 5-5 \cdot 7)$ & $2 \cdot 8(0 \cdot 9-8 \cdot 1)$ & $2 \cdot 1(0 \cdot 6-6 \cdot 8)$ \\
\hline Past history of emotional problems & NA & $6 \cdot 4(1 \cdot 2-34 \cdot 3)$ & $1 \cdot 4(0 \cdot 4-4 \cdot 6)$ & $3 \cdot 0(0 \cdot 8-11 \cdot 5)$ \\
\hline Does regular sport & $1 \cdot 1(0 \cdot 3-4 \cdot 1)$ & $0 \cdot 9(0 \cdot 3-2 \cdot 8)$ & $0 \cdot 7(0 \cdot 2-2 \cdot 1)$ & $1 \cdot 1(0 \cdot 4-3 \cdot 4)$ \\
\hline \multicolumn{5}{|l|}{ Features of glandular fever at baseline assessment } \\
\hline$\geqslant 10$ days off sick & NA & $4 \cdot 0(1 \cdot 1-14 \cdot 4)$ & $1 \cdot 1(0 \cdot 3-3 \cdot 8)$ & $1 \cdot 2(0 \cdot 3-4 \cdot 9)$ \\
\hline 'Recovered' at interview & $0 \cdot 5(0 \cdot 1-2 \cdot 0)$ & $0 \cdot 2(0 \cdot 3-0 \cdot 9)$ & $0 \cdot 9(0 \cdot 3-2 \cdot 7)$ & $0 \cdot 6(0 \cdot 2-2 \cdot 3)$ \\
\hline Given advice to rest by GP & $0 \cdot 6(0 \cdot 2-2 \cdot 5)$ & $0 \cdot 6(0 \cdot 2-1 \cdot 9)$ & $1 \cdot 3(0 \cdot 4-3 \cdot 9)$ & $0 \cdot 8(0 \cdot 2-2 \cdot 7)$ \\
\hline High total symptom* & $4 \cdot 4(1 \cdot 0-18)$ & $8 \cdot 6(2 \cdot 0-37)$ & $1 \cdot 6(0 \cdot 5-4 \cdot 7)$ & $5 \cdot 7(1 \cdot 4-24)$ \\
\hline High symptoms severity* & $14(1 \cdot 7-118)$ & $12(2 \cdot 8-52)$ & $1 \cdot 8(0 \cdot 6-5 \cdot 3)$ & $4 \cdot 8(1 \cdot 8-27)$ \\
\hline Low physical functioning (SF-36)* & $12(1 \cdot 5-101)$ & $4 \cdot 5(1 \cdot 3-15)$ & $1 \cdot 0(0 \cdot 4-2 \cdot 8)$ & $1 \cdot 5(0 \cdot 5-4 \cdot 6)$ \\
\hline Poor physical functioning in last month (SF-36)* & $8.0(1.9-34)$ & $6 \cdot 7(1 \cdot 6-28)$ & $1 \cdot 3(0 \cdot 4-4 \cdot 3)$ & $1 \cdot 1(0 \cdot 3-4 \cdot 1)$ \\
\hline Poor emotional functioning last month (SF-36)* & $4 \cdot 5(0 \cdot 9-22 \cdot 6)$ & $2 \cdot 9(0 \cdot 9-9 \cdot 4)$ & $1 \cdot 3(0 \cdot 5-3 \cdot 5)$ & $0 \cdot 8(0 \cdot 2-2 \cdot 5)$ \\
\hline Poor functioning on WSAS* & $17(2 \cdot 1-144)$ & $20(3 \cdot 8-1 \cdot 4)$ & $2 \cdot 7(0 \cdot 9-8 \cdot 0)$ & $6 \cdot 5(1 \cdot 5-27)$ \\
\hline GHQ- $12>5^{*}$ & $6 \cdot 3(1 \cdot 3-32)$ & $11(2 \cdot 7-42)$ & $2 \cdot 8(1 \cdot 0-8 \cdot 1)$ & $1 \cdot 1(0 \cdot 4-3 \cdot 6)$ \\
\hline Low control/cure (IPQ)* & $0 \cdot 3(0 \cdot 1-1 \cdot 6)$ & $1 \cdot 6(0 \cdot 5-5 \cdot 1)$ & $0 \cdot 6(0 \cdot 2-1 \cdot 6)$ & $1 \cdot 7(0 \cdot 5-5 \cdot 9)$ \\
\hline High consequences (IPQ)* & $4 \cdot 0(1 \cdot 0-17)$ & $5 \cdot 8(1 \cdot 6-20)$ & $3 \cdot 0(1 \cdot 0-9 \cdot 0)$ & $1 \cdot 4(0 \cdot 4-4 \cdot 5)$ \\
\hline Expect recovery will take $>1$ month & NA & $8 \cdot 4(2 \cdot 0-36)$ & $3 \cdot 4(1 \cdot 1-11)$ & $3 \cdot 0(0 \cdot 9-11)$ \\
\hline
\end{tabular}

* Indicates median split used; NA, odds ratio not presented due to empty cells.

SF-36, Short Form 36; WSAS, Work and Social Adjustment Scale; GHQ-12, General Health Questionnaire; IPQ, Illness Perceptions Questionnaire.

Statistically significant associations $(P<0 \cdot 05)$ are shown in bold type.

Table 4. Results of factor analysis

\begin{tabular}{lcc}
\hline \hline Variable & $\begin{array}{c}\text { Symptoms- } \\
\text { disability }\end{array}$ & $\begin{array}{c}\text { Fatigue- } \\
\text { depression }\end{array}$ \\
\hline SF-36 Physical functioning & $-0 \cdot 74^{*}$ & \\
SF-36 Physical functioning & & \\
SF-36 Emotional function & $0 \cdot 77$ & \\
WSAS & $0 \cdot 41$ & $0 \cdot 60$ \\
Baseline fatigue & 0.67 & $0 \cdot 41$ \\
Severity of physical & & 0.66 \\
symptoms & & \\
GHQ score & & \\
\hline
\end{tabular}

WSAS, Social and Work Adjustment Scale; GHQ, General Health Questionnaire; SF-36, Short form 36.

Results only shown if rotated factor loading $>0 . *$ SF-36 factor loading is negative because high score indicates good functioning.

and that the illness would have more serious consequences (IPQ subscale)). By 12 months, the main predictor was increasing age, female geneder and symptoms-disability at baseline; illness perceptions were no longer associated. Similar analyses were performed using multiple regression analysis, with fatigue as a continuous outcome as the dependent variable. This yielded similar results (results not shown).

\section{DISCUSSION}

Our results indicate that fatigue at 3 months is strongly predicted by the severity of baseline symptoms, the belief that the illness will take $>1$ month to recover from, and immune activation. By 6 months, the main predictors are illness perceptions at baseline, but at 12 months older age and female gender are the key predictors, as well as a weaker effect of baseline symptoms. At no point were cortisol levels related to fatigue.

Before addressing these findings in more detail it is necessary to make some general comments on the methodology. First, this was a pilot study with a small sample size, which may have resulted in type two errors. For example, the association between immune activation and fatigue at 3 months, but not later, does not rule out the possibility of an association with later fatigue. Secondly, the follow-up rates at 3 and 12 months were disappointing. The most robust findings were those at 6 months where the best follow-up was achieved. Thirdly, the results were taken from within a randomized controlled trial, and it is possible that the intervention itself 
Table 5. Results of logistic regression analysis

\begin{tabular}{|c|c|c|c|}
\hline \multirow[b]{2}{*}{ Variable } & \multicolumn{3}{|c|}{ Fatigue case at } \\
\hline & 3 months $(N=49)$ & 6 months $(N=62)$ & 12 months $(N=50)$ \\
\hline \multicolumn{4}{|l|}{ Step 1 variables } \\
\hline Age & & & $1 \cdot 10(1 \cdot 00-1 \cdot 21)$ \\
\hline Female gender & & $4 \cdot 54(1 \cdot 23-16 \cdot 8)$ & $4 \cdot 36(0 \cdot 98-19 \cdot 5)$ \\
\hline \multicolumn{4}{|l|}{ Step 2 variables } \\
\hline Symptoms-disability & $3 \cdot 11(1 \cdot 35-7 \cdot 17)$ & & $2 \cdot 22(0 \cdot 99-5 \cdot 02)$ \\
\hline Fatigue-depression & $3.73(1.41-9.91)$ & & \\
\hline \multicolumn{4}{|l|}{ Step 3 variables } \\
\hline Recovery will take $>1$ month & $7 \cdot 60(1 \cdot 11-52)$ & $4 \cdot 20(1 \cdot 11-16 \cdot 0)$ & \\
\hline IPQ consequences & & $1 \cdot 18(1.01-1 \cdot 37)$ & \\
\hline
\end{tabular}

Parameters are ORs and $95 \%$ confidence intervals. For the continuous variables (age, symptoms-disability, fatigue-depression and IPQ consequences) results indicate the increase in odds associated with each unit increase in the variable.

affected some of our key predictor variables such as illness perceptions. We report the results for the randomized controlled trial separately (Candy et al. 2003), and we have controlled for any effect of the intervention in the multivariate analyses shown here. The intervention would only bias the results of the present study if there were interactions between the predictors described here and the intervention. We are unable to test for these interactions due to inadequate statistical power.

\section{Immune variables}

Our findings are not dissimilar to those of White et al. (2001) where an association, during the acute illness phase, between total CD8 T cell count and fatigue levels was seen, although no correlations were found with total CD4 and fatigue. In the present study we chose to measure the activated subsets of CD4 and CD8 T cells, rather than those of the total populations. We reasoned that these subsets would be more reflective of the degree of immune activation, and therefore have a stronger relationship with acute, 'immune-mediated' fatigue. This indeed proved to be the case, with both activated CD4 and CD8 subsets being associated with acute fatigue. Like White and colleagues, we did not find any evidence that these immune changes led to longer-term fatigue, but due to the small samples in which we had immune data, and some losses to follow-up this could have been a type II error.

\section{Cortisol}

Our study failed to show any relationship between fatigue and low cortisol. There was no link between cortisol levels and fatigue at 0,3 or 6 months, and those with lower cortisol levels at baseline were no more likely to go on to develop fatigue than those with higher levels. There is now accumulating evidence that patients with established CFS do have impaired function of the adrenal axis on some measures (Cleare $e t$ al. 2001; Parker et al. 2001). Most of these studies have been undertaken on participants ill for several years or longer, with more severe disability, and with more strictly defined CFS. Our results suggest that abnormalities in cortisol may not occur early on in the genesis of chronic fatigue, and are not a risk factor for chronic fatigue of 6 months duration. It is perhaps more likely that other factors, perhaps those secondary to prolonged fatigue and disability, are responsible for the observed changes in adrenal function in those more chronically and severely ill. Alternatively, those destined to go on to develop more severe and chronic illness may indeed have impaired adrenal function but were too uncommon to have been detected in this small study.

\section{Illness perceptions}

In the multivariate analysis we found that expectations that the individual would take a long time to recover, or that the illness would have serious consequences were associated with a poorer outcome up to 6 months later. This was not explained by symptom severity during the acute phase.

Two previous cohort studies have explored fatigue following the onset of IM. Buchwald and colleagues (2000) found self-report of 
non-recovery at 2 months was best determined by biological factors, whereas the 6 months predictors represented a more complex mix of psychological and social factors. In White and colleagues' cohort the most reliable predictor of fatigue syndrome to 6 months after onset was poorer physical fitness (White et al. 2001), a variable we did not test in the present study. These studies explored different predictors and outcomes, but like us they both found that the predictors of poor outcome depended on when the outcome was measured. Acute or subacute fatigue seems to have different predictors, possibly more strongly related to the severity of the original illness, than chronic fatigue.

Out study is the first to explore initial illness perceptions and subsequent recovery in IM. The importance of illness perceptions as predictors of poorer health outcomes has been found for other conditions (Sharloo et al. 1998; Fortune et al. 2000; Edwards et al. 2001). We now extend this to IM. The testing of interventions aimed at increasing beliefs in personal control and changing beliefs about the course and the consequences of the illness to help improve outcome is now underway for various conditions (Clark \& Hampson, 2001; Petrie et al. 2002). The trial of an intervention to aid recovery in IM that aimed at promoting certain perceptions and as well as advising a time-targeted return to normal activity has been tested by us in an exploratory study and found to improve medium-term fatigue levels (to be reported elsewhere). Such as intervention should be tested in larger randomized controlled trials in IM patients prior to general implementation.

This study was funded by the Linbury Trust. We thank Dr Andrew Papadopoulos for undertaking salivary cortisol assays, and Lucia Poon, the participating GPs, and staff from the virology and haematology laboratories involved for their assistance in recruiting patients.

\section{REFERENCES}

Berelowitz, G. J., Burgess, A. P., Thanabalasingham, T., MurrayLyon, I. M. \& Wright, D. J. M. (1995). Post-hepatitis syndrome revisited. Journal of Viral Hepatitis 2, 133-138.

Buchwald, D., Rea, T. D., Katon, W. J., Russo, J. E. \& Ashley, R. L. (2000). Acute infectious mononucleosis: characteristics of patients who report failure to recover. American Journal of Medicine 109, 531-537.

Candy, B., Chalder, T., Cleare, A., White, P. D., Wessely, S. \& Hotopf, M. (2003). Recovery from glandular fever: a case for more than symptomatic therapy? A systematic review. Journal of Psychosomatic Research (submitted).

Chalder, T., Berelowitz, C. \& Pawlikowska, T. (1993). Development of a fatigue scale. Journal of Psychosomatic Research 37, 147-154.

Clark, M. \& Hampson, S. E. (2001). Implementing a psychological intervention to improve lifestyle self-management in patients with type 2 diabetes. Patient Education and Counselling 42, 247-256.

Cleare, A. J., Blair, D., Chambers, S. \& Wessely, S. (2001). Urinary free cortisol in chronic fatigue syndrome. American Journal of Psychiatry 158, 641-643.

Cochrane, R. \& Robertson, A. (1973). The life events inventory: a measure of the relative severity of psycho-social stressors. Journal of Psychosomatic Research 17, 135-139.

Cope, H., David, A., Pelosi, A. \& Mann, A. (1994). Predictors of chronic 'postviral' fatigue. Lancet 344, 864-868.

Edwards, R., Suresh, R., Lynch, S., Clarkson, P. \& Stanley, P. (2001). Illness perceptions and mood in chronic fatigue syndrome. Journal of Psychosomatic Research 50, 65-68.

Fortune, D. G., Richards, H. L., Min, C. J. \& Griffiths, C. E. M. (2000). Pathological worrying, illness perceptions and disease severity in patients with psoriasis. British Journal of Health Psycho$\log y$ 5, 71-82.

Garratt, A., Ruta, D., Abdalla, M., Buckingham, J. \& Russell, I. (1993). The SF-36 health survey questionnaire: an outcome measure suitable for use within the NHS? British Medical Journal 306, 1440-1444.

Goldberg, D. \& Williams, P. (1988). A User's Guide to the General Health Questionnaire. NFER-Nelson: Windsor.

Hotopf, M., Mayou, R., Wadsworth, M. \& Wessely, S. (1999). Childhood risk factors for adult medically unexplained symptoms: results of a national birth cohort study. American Journal of Psychiatry 156, 1796-1800.

Hotopf, M., Wilson-Jones, C., Mayou, R., Wadsworth, M. \& Wessely, S. (2000). Childhood predictors of adult medically unexplained hospitalisations. Results from a national birth cohort study. British Journal of Psychiatry 176, 273-280.

Hotopf, M. H., Noah, N. \& Wessely, S. (1996). Chronic fatigue and psychiatric morbidity following viral meningitis: a controlled study. Journal of Neurology Neurosurgery and Psychiatry 60, 504-509.

Leese, G., Chattington, P., Fraser, W., Vora, J., Edwards, R. \& Williams, G. (1996). Short-term night-shift working mimics the pituitary-adrenocortical dysfunction of chronic fatigue syndrome. Journal of Clinical Endocrinology and Metabolism 81, 1867-1870.

Marks, I. (1986). Behavioural Psychotherapy: Maudsley Pocket Book of Clinical Management. Wright: Bristol.

Parker, A., Wessely, S. \& Cleare, A. J. (2001). The neuroendocrinology of chronic fatigue syndrome and fibromyalgia. Psychological Medicine 31, 1331-1345.

Petrie, K. J., Cameron, L. D., Ellis, C. J., Buick, D. \& Weinman, J. (2002). Changing illness perceptions following myocardial infarction: an early intervention randomised controlled trial. Psychosomatic Medicine (in the press).

Power, M., Champion, L. A. \& Aris S. J. (1988). The development of a measure of social support: the Significant Others Scale (SOS) British Journal of Clinical Psychology 27, 349-358.

Sharloo, M., Kaptein, A. A., Weinman, J., Hazes, J. M., Willems, L. N. A., Bergman, W. \& Rooijmans, H. G. M. (1998). Illness perceptions, coping and functioning in patients with rheumatoid arthritis, chronic obstructive pulmonary disease and psoriasis. Journal of Psychosomatic Research 44, 573-585.

Spath Schwalde, E., Scholle, T., Kern, W., Fehm, H. L. \& Born, J. (1992). Nocturnal adrenocorticotrophin and cortisol secretion depends on sleep duration and decreases in association with spontaneous awakening in the morning. Journal of Clinical Endocrinology and Metabolism 75, 1431-1435.

Stupnicki, R. \& Obminski, Z. (1992). Glucocorticoid response to exercise as measured by serum and salivary cortisol. European Journal of Applied Physiology 65, 546-549.

Weinman, J., Petrie, K. J., Moss-Morris, R. \& Horne, R. (1996). The Illness Perception Questionnarie: a new method for assessing the 
cognitive representation of illness. Psychology and Health 11, 431-445.

Wessely, S., Hotopf, M. \& Sharpe, M. (1998). Chronic Fatigue and Its Syndromes. Oxford University Press: Oxford.

White, P. D., Thomas, J. M., Amess, J., Crawford, D. H., Grover, S. Kangro, H. \& Clare, A. (1998). Incidence, risk and prognosis of acute and chronic fatigue syndromes and psychiatric disorders after glandular fever. British Journal of Psychiatry 173, 475-481.

White, P. D., Thomas, J. M., Kangro, H., Bruce-Jones, W. D. A., Amess, J., Crawford, D. H., Grover, S. A. \& Clare, A. W. (2001). Predictions and association of fatigue syndromes and mood disorders that occur after infectious mononucleosis. Lancet 358, $1946-1954$. 